\title{
CORONAL DISCONNECTION EVENTS AND METRIC RADIO EMISSION
}

\author{
N. GOPALSWAMY \\ Dept. of Astronomy, University of Maryland, College Park, MD 207.12 USA
}

\begin{abstract}
.
We review the existing literature on the coronal disconnection events (CDEs) and discuss the importance of these events in understanding coronal structures. We discuss the possible radio signatures of the CDEs and how they may be observed by radio instruments.
\end{abstract}

Key words: Sun - CME - Disconnection - Radio emission

\section{Introduction}

Coronal Disconnection Events (CDEs) represent a class of coronal mass ejections (CMEs) in which open or closed magnetic structures detach from the sun and propagate into the interplanetary medium. The CDEs seem to leave behind a closed field structure anchored to the Sun as compared to the total eruption in the case of regular CMEs. In the coronagraph images, the CDEs are seen as $\mathrm{U}$ or $\mathrm{V}$ shaped structures moving into the corona. These structures have a 'concave-outward' geometry as opposed to the regular CMEs structures which are concave toward the Sun. Some of the motivations to study the CDEs: The CDE (i) may be important in deciding the topology of the interplanetary magnetic field; (ii) may serve as a direct evidence of magnetic reconnection; and (iii) may help maintain a constant level of interplanetary magnetic flux as observed. It was initially thought that the CDEs are rare, but now they seem to be quite significant in number $(10 \%$ of all CMEs may be CDEs - Webb 1993 private communication). To our knowledge there are only three studies reported in the literature and bence the CDEs represent a poorly studied class of CMEs. Two of these (11ling and Hundhausen, 1983; McComas et al 1991) dealt with only coronagraph observations while the third (Gopalswamy et al. 1994) reported detection of metric radio continuum in association with a CDE. Cliver (1989) has also reported on CDEs based on old eclipse observations. The aim of this paper is to explore the possible radio signatures of the CDEs that one expects to observe at metric radio wavelengths. 


\section{Summary of Observations of CDEs}

Iling and Hundhausen (1983) presented the first evidence of a disconnection event following a CME in the SMM-C/P field of view. The detached structure moved out with a speed of about $175 \mathrm{kms}^{-1}$. This speed was 3 - 4 times larger than that of the preceding C.ME. Iling and Hundhausen (1983) interpreted the $U$ shaped feature as a compressional front behind the expelled closed loop. The region of reconnection was estimated to be below the oculting disk of the SMM-C/P $\left(1.6 R_{\odot}\right)$. McComas et al (1991) reported a CDE where previously open, oppositely directed field lines were supposed to have reconnected to form the U shaped feature. The reconnection, according to these authors, could have been initiated by compressional waves propagating in the lateral direction. A parabolic fit to the height time history of the $U$ shaped feature yielded a height of the disconnection as $1.7 R_{\odot}$ which is clearly above the SMM-C/P's occulting disk. The U shaped feature showed an acceleration of $6.2 \mathrm{~ms}^{-2}$ and a speed of $\sim 170 \mathrm{kms}^{-1}$ at a height of $5 R_{\odot}$. The event reported by Gopalswamy et al. (1994) was very similar to the McComas et al. (1991) event in that it had similar starting height and structure. The acceleration and speed, however, were relatively larger. A nonthermal continuum was (observed by the Clark Lake radioheliograph) located radially below the trajectory of the U shaped structure (see Fig. 1). The radio continuum was observed at $73.8,50$ and $38.5 \mathrm{MHz}$ and was found to have bursty time structure, indicative of repeated injection of nonthermal particles. From the observed time structure, Gopalswamy et al. (1994) derived an energy release rate $\left(1.3 \times 10^{-24} \mathrm{ergs}^{-1}\right)$, smaller than that during flare emissions. This is at present the only event that detected radio emission in association with a CDE; if confirmed using further observations, this will prove to be an important evidence for magnetic reconnection and particle acceleration.

\section{Radio Signatures of CDEs}

In Fig. 2 we have shown a cartoon summarizing possible structures that could be expelled from the Sun in order to illustrate the expected radio signatures of the disconnection event. The detachment of a closed (open) field structure results in a set of stationary loops connected to the solar surface and moving structure which is closed (open). Since closed field lines can trap and accumulate energetic particles accelerated during the reconnection process, the ejection of a closed field structure results in two continuum radio sources, one from the loops connected to the solar surface and the other from the moving structure; open moving structures can not trap particles and hence cannot produce continuum radio sources. Moving type IV burst 


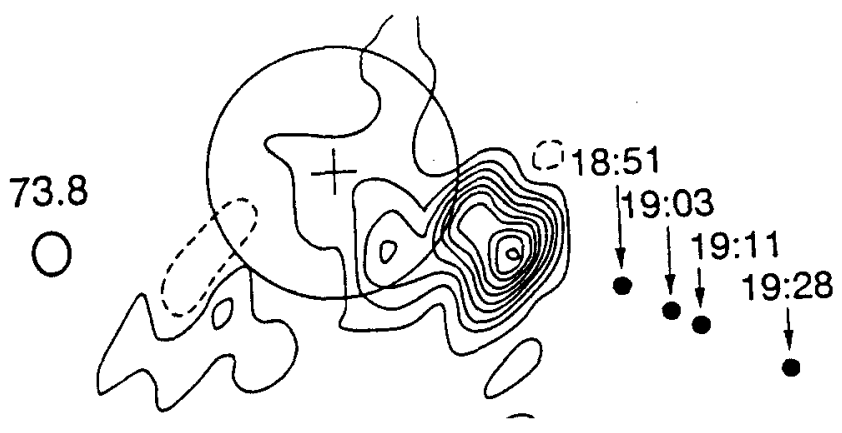

Fig. 1. Superposition of $73.8 \mathrm{MHz}$ radio contours on the trajectory of U shaped feature during the 1986 Februray $13 \mathrm{CDE}$. The large circle represents the optical Sun. The inner source in the radio map is due to a long lasting noise storm. The $73.8 \mathrm{MHz}$ beam is shown at the left. The contour levels are at 5 to $95 \%$ of the peak brightness temperature $(4.2$ $\mathrm{MK})$ in steps of $10 \%$. The filled circles represent the positions of the $U$ shaped feature at the marked times.

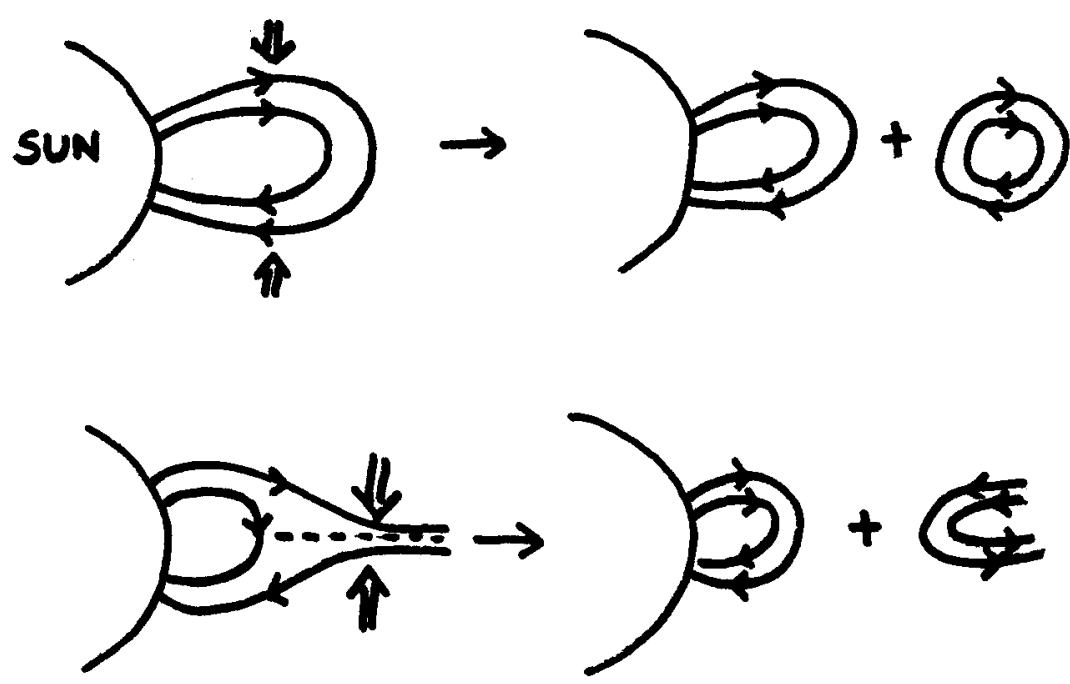

Fig. 2. Schematic diagram showing the two possible structures that can be expelled during a CDE. The actual structure is expected to be three dimensional. 'The double arrows in the vertical direction represent lateral compression.

of the isolated source variety is similar to the case of detached closed field structure, although it is believed to be formed out of an erupting prominence (Gopalswamy and $K u n d u, 1990$ ). If the radio instrument is sensitive enough, it may be possible to observe type III-like bursts along the detaching 
open field structures as the accelerated particles escape along the open field lines. We must point out that the CDEs occur at large heights in the corona. Most of the ground based radio instruments may not have access to these heights and one may have to use spaceborne instruments to detect the radio emissions. In summary the CDEs may result in one or more of the following: stationary continuum, moving continuum and type III-like bursts.

\section{Discussion and Conclusions}

1. All the CDEs studied so far occurred following a CME. This may suggest that the disturbed conditions prevailing behind a CME may be needed for a CDE to take place. It is necessary to study more events to decide if the preceding $\mathrm{CME}$ is essential for a $\mathrm{CDE}$. One interesting question may be whether the disconnection takes place in long lasting streamer structures without a "streamer disruption".

2. The $U$ shaped structure may represent open field structures or it may simply be a compressional front behind the expelled closed field structure (Illing and Hundhausen, 1983). Several CME observations have shown blobs of bright material ejected but it is not clear if these are detached structures (see e.g. Gergely et al 1984).

3. Radio instruments operating at metric or lower frequencies may be able to detect radio signatures of CDEs in the form of stationary and moving continua and type III-like bursts.

\section{Acknowledgements}

The author thanks the American Astronomical Society and the International Astronomical Union for their support to attend the IAU Colloquium 154 where this paper was presented. This research was supported by grants from the National Science Foundation and NASA to the University of Maryland at College Park.

\section{References}

Cliver, E. W.: 1989, Solar Phys. 122, 319

Gergely, T. E. et al.: 1984, Solar Phys. 90, 161

Gopalswamy, N. and Kundu, M. R.: 1990, Astrophys. $J$ 365, L31

Gopalswamy, N., Kundu, M. R. and St. Cyr, O. C.: 1994, Astrophy.s. J 424, L135

Illing, R. M. E. and Hundhausen, A. J. : 1983, J. Geophys. Res. 88, 10210

McComas, D. J., Phillips, J. L., Hundhausen, A. J. and Burkpile, J. T.: 1991, Geophys. Res. Lett. 18, 73

Webb, D.: 1993, , private communication 